\title{
Fibonacci-like behavior of the number of numerical semigroups of a given genus
}

\author{
Maria Bras-Amorós * \\ Published at \\ Semigroup Forum, Springer, vol. 76, n. 2, pp. 379-384, March 2008
}

\begin{abstract}
We conjecture a Fibonacci-like property on the number of numerical semigroups of a given genus. Moreover we conjecture that the associated quotient sequence approaches the golden ratio. The conjecture is motivated by the results on the number of semigroups of genus at most 50 . The Wilf conjecture has also been checked for all numerical semigroups with genus in the same range.
\end{abstract}

\section{Introduction}

Let $\mathbb{N}_{0}$ denote the set of all non-negative integers. A numerical semigroup is a subset $\Lambda$ of $\mathbb{N}_{0}$ containing 0 , closed under addition and with finite complement in $\mathbb{N}_{0}$. For a numerical semigroup $\Lambda$ define the genus of $\Lambda$ as the number $g=\#\left(\mathbb{N}_{0} \backslash \Lambda\right)$. As an example of numerical semigroup one can take

$$
\{0,4,5,8,9,10\} \cup\left\{i \in \mathbb{N}_{0}: i \geqslant 12\right\} .
$$

In this case the genus is 6 .

We are interested on the number $n_{g}$ of numerical semigoups of genus $g$. It is obvious that $n_{0}=1$ since $\mathbb{N}_{0}$ is the unique numerical semigroup of genus 0 . On the other hand, if 1 is in a numerical semigroup, then any non-negative integer must belong also to the numerical semigroup, because any non-negative integer is a finite sum of 1's. Thus, the unique numerical semigroup with genus 1 is $\{0\} \cup\left\{i \in \mathbb{N}_{0}: i \geqslant 2\right\}$ and $n_{1}=1$. We conjecture

1. $n_{g} \geqslant n_{g-1}+n_{g-2}$, for $g \geqslant 2$

2. $\lim _{g \rightarrow \infty} \frac{n_{g-1}+n_{g-2}}{n_{g}}=1$

3. $\lim _{g \rightarrow \infty} \frac{n_{g}}{n_{g-1}}=\phi$, where $\phi$ is the golden ratio.

\footnotetext{
*Universitat Rovira i Virgili, Catalonia, Spain, e-mail: maria.bras@urv.com
} 
Notice that point 2 would mean the sequence $n_{g}$ to behave asymptotically as the Fibonacci sequence. This conjecture is motivated by the results on the number of semigroups of genus at most 50 .

\section{Computation of $n_{g}$}

Every numerical semigroup can be generated by a finite set of elements and a minimal set of generators is unique (see for instance [3]). Let $m$ be the smallest non-zero element of a numerical semigroup $\Lambda$, usually referred as its multiplicity. The set of minimal generators of $\Lambda$ can be easily computed because it is a subset of the finite set

$$
\{m\} \cup\{\lambda \in \Lambda \backslash\{0\}: \lambda-m \notin \Lambda\} .
$$

This last set is intimately related to the Apéry set of $\Lambda$ [1, 3, 5, 6, 4,

The conductor of a numerical semigroup $\Lambda$ is the unique integer $c$ with $c-1 \notin \Lambda$ and $c+\mathbb{N}_{0} \subseteq \Lambda$. Given a numerical semigroup $\Lambda$ of genus $g$ and conductor $c, \Lambda \cup\{c-1\}$ is a numerical semigroup and its genus is $g-1$. So, any numerical semigroup of genus $g$ can be obtained from a numerical semigroup of genus $g-1$ by removing one element larger than or equal to its conductor. It is easy to check that when removing such an element from a numerical semigroup, the set obtained is a numerical semigroup if and only if the removed element belongs to the set of minimal generators.

For instance, the unique numerical semigroup of genus 0 is $\mathbb{N}_{0}$. Its unique minimal generator is 1 . Now, removing 1 from $\mathbb{N}_{0}$ we obtain

$$
\Lambda_{1}=\{0\} \cup\left\{i \in \mathbb{N}_{0}: i \geqslant 2\right\} .
$$

The numerical semigroup $\Lambda_{1}$ is the unique numerical semigroup of genus 1 . In turn, $\Lambda_{1}$ has minimal generators 2 and 3 . By removing 2 from $\Lambda_{1}$ we obtain the numerical semigroup

$$
\Lambda_{2,1}=\{0\} \cup\left\{i \in \mathbb{N}_{0}: i \geqslant 3\right\}
$$

and by removing 3 from $\Lambda_{1}$ we obtain the numerical semigroup

$$
\Lambda_{2,2}=\{0,2\} \cup\left\{i \in \mathbb{N}_{0}: i \geqslant 4\right\} .
$$

The semigroups $\Lambda_{2,1}$ and $\Lambda_{2,2}$ are all the numerical semigroups of genus 2 .

For the results in this paper we computed $n_{g}$ by brute approach. That is, we generated all numerical semigroups of genus $g$ from all numerical semigroups of genus $g-1$ as explained and then we counted them. This way one should be able to generate all semigroups of any given genus.

In the web page http://w3.impa.br/〜nivaldo/algebra/semigroups/index.html by Nivaldo Medeiros one can find all numerical semigroups of genus up to 12. In Neil Sloane's On-line Encyclopedia of Integer Sequences [7] there are the values of $n_{g}$ for $g \leqslant 14$. We could compute all numerical semigroups of genus up to 50 . 


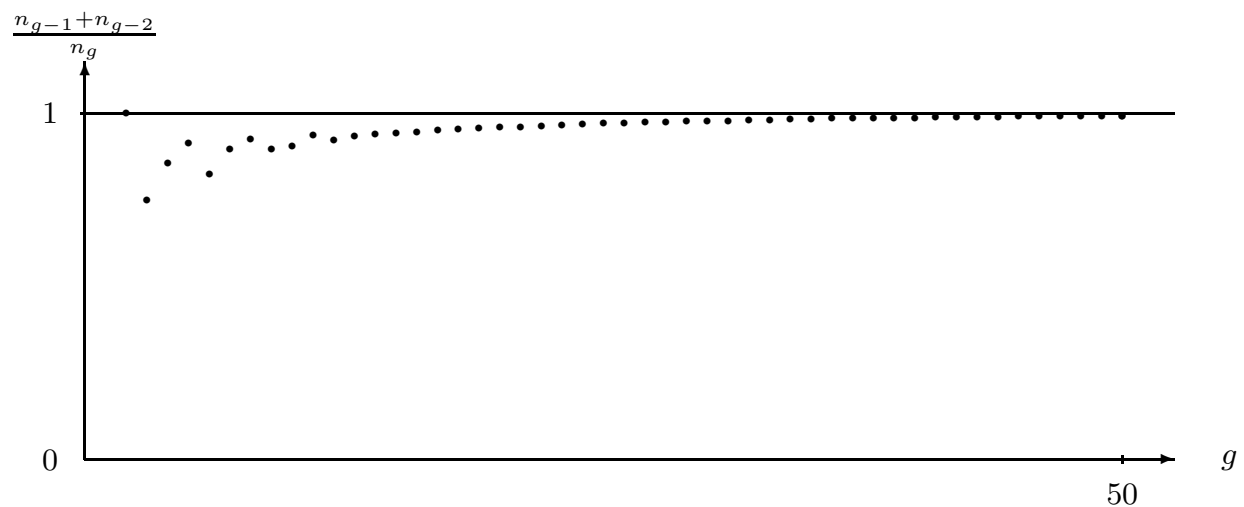

Figure 1: Behavior of the quotient $\frac{n_{g-1}+n_{g-2}}{n_{g}}$. The values in this graphic correspond to the values in Table 1 .

The obstruction on the calculus of all numerical semigroups of a given large genus using the method explained above is the huge size of the results and the need to keep them for the next step. Indeed, the growth of $n_{g}$ is apparently exponential with $g$ and doing computations beyond a certain genus is really difficult using the computational means one can find at present. For instance, using a Pentium D $3.00 \mathrm{GHz}$ with $1 \mathrm{~GB}$ of RAM it took 19 days to compute all semigroups of genus 50 and we expect that it would take about one month to compute all numerical semigroups of genus 51 . The size of the compressed file containing all numerical semigroups of genus 50 is $3.6 \mathrm{~GB}$.

In Table 1 there are the results obtained for all numerical semigroups with genus up to 50. For each genus we wrote the number of numerical semigroups of the given genus, the Fibonacci-like-estimated value given by the sum of the number of semigroups of the two previous genus, the value of the quotient $\frac{n_{g-1}+n_{g-2}}{n_{g}}$, and the value of the quotient $\frac{n_{g}}{n_{g-1}}$. In Figure 1 and Figure 2 we depicted the behavior of these quotients. From these graphics one can predict that $\frac{n_{g-1}+n_{g-2}}{n_{g}}$ approaches 1 as $g$ approaches infinity whereas $\frac{n_{g}}{n_{g-1}}$ approaches the golden ratio as $g$ approaches infinity. We leave this as a conjecture.

\section{On the Wilf conjecture}

The Wilf conjecture ([8, 2]) states that the number $e$ of minimal generators of a numerical semigroup of genus $g$ and conductor $c$ satisfies

$$
e \geqslant \frac{c}{c-g}
$$

It is easy to check it when the numerical semigroup is symmetric, that is, when $c=2 g$. In [2] the inequality is proved for many other cases. Here we proved by 


\begin{tabular}{|ccccc|}
\hline$g$ & $n_{g}$ & $n_{g-1}+n_{g-2}$ & $\frac{n_{g-1}+n_{g-2}}{n_{g}}$ & $\frac{n_{g}}{n_{g-1}}$ \\
\hline 0 & 1 & & & \\
1 & 1 & & & 1 \\
2 & 2 & 2 & 1 & 2 \\
3 & 4 & 3 & 0.75 & 2 \\
4 & 7 & 6 & 0.857143 & 1.75 \\
5 & 12 & 11 & 0.916667 & 1.71429 \\
6 & 23 & 19 & 0.826087 & 1.91667 \\
7 & 39 & 35 & 0.897436 & 1.69565 \\
8 & 67 & 62 & 0.925373 & 1.71795 \\
9 & 118 & 106 & 0.898305 & 1.76119 \\
10 & 204 & 185 & 0.906863 & 1.72881 \\
11 & 343 & 322 & 0.938776 & 1.68137 \\
12 & 592 & 547 & 0.923986 & 1.72595 \\
13 & 1001 & 935 & 0.934066 & 1.69088 \\
14 & 1693 & 1593 & 0.940933 & 1.69131 \\
15 & 2857 & 2694 & 0.942947 & 1.68754 \\
16 & 4806 & 4550 & 0.946733 & 1.68218 \\
17 & 8045 & 7663 & 0.952517 & 1.67395 \\
18 & 13467 & 12851 & 0.954259 & 1.67396 \\
19 & 22464 & 21512 & 0.957621 & 1.66808 \\
20 & 37396 & 35931 & 0.960825 & 1.66471 \\
21 & 62194 & 59860 & 0.962472 & 1.66312 \\
22 & 103246 & 99590 & 0.964589 & 1.66006 \\
23 & 170963 & 165440 & 0.967695 & 1.65588 \\
24 & 282828 & 274209 & 0.969526 & 1.65432 \\
25 & 467224 & 453791 & 0.971249 & 1.65197 \\
26 & 770832 & 750052 & 0.973042 & 1.64981 \\
27 & 1270267 & 1238056 & 0.974642 & 1.64792 \\
28 & 2091030 & 2041099 & 0.976121 & 1.64613 \\
29 & 3437839 & 3361297 & 0.977735 & 1.64409 \\
30 & 5646773 & 5528869 & 0.97912 & 1.64254 \\
31 & 9266788 & 9084612 & 0.980341 & 1.64108 \\
32 & 15195070 & 14913561 & 0.981474 & 1.63973 \\
33 & 24896206 & 24461858 & 0.982554 & 1.63844 \\
34 & 40761087 & 40091276 & 0.983567 & 1.63724 \\
35 & 66687201 & 65657293 & 0.984556 & 1.63605 \\
36 & 109032500 & 107448288 & 0.98547 & 1.63498 \\
37 & 178158289 & 175719701 & 0.986312 & 1.63399 \\
38 & 290939807 & 287190789 & 0.987114 & 1.63304 \\
39 & 474851445 & 469098096 & 0.987884 & 1.63213 \\
40 & 774614284 & 765791252 & 0.98861 & 1.63128 \\
41 & 1262992840 & 1249465729 & 0.98929 & 1.63048 \\
42 & 2058356522 & 2037607124 & 0.989919 & 1.62975 \\
43 & 3353191846 & 3321349362 & 0.990504 & 1.62906 \\
44 & 5460401576 & 5411548368 & 0.991053 & 1.62842 \\
45 & 8888486816 & 8813593422 & 0.991574 & 1.62781 \\
46 & 14463633648 & 14348888392 & 40.992067 & 1.62723 \\
47 & 23527845502 & 23352120464 & 0.992531 & 1.62669 \\
48 & 38260496374 & 37991479150 & 0.992969 & 1.62618 \\
49 & 62200036752 & 61788341876 & 0.993381 & 1.6257 \\
50 & 101090300128 & 100460533126 & 0.99377 & 1.62525 \\
\hline & & & & \\
& & & &
\end{tabular}

Table 1: Computational results on the number of numerical semigroups up to genus 50 . 


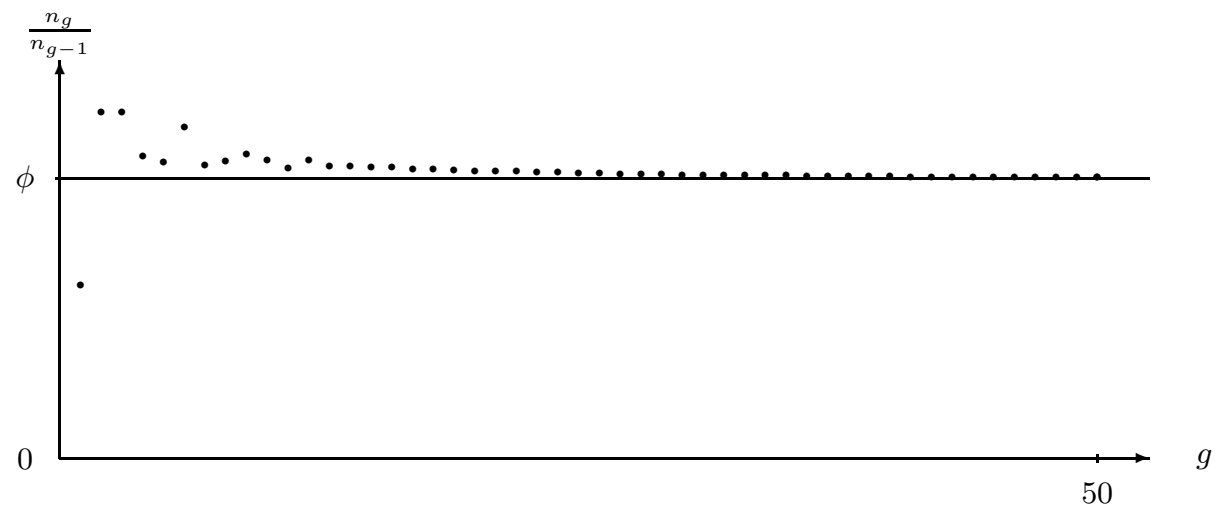

Figure 2: Behavior of the quotient $\frac{n_{g}}{n_{g-1}}$. The values in this graphic correspond to the values in Table 1.

brute approach that any numerical semigroup of genus at most 50 also satisfies the conjecture.

\section{Acknowledgments}

The author would like to thank Jordi Funollet and Josep M. Mondelo for their help on the computations and Pedro A. García-Sánchez, José Carlos Rosales, Aureliano M. Robles, and Ruud Pellikaan for many valuable discussions.

This work was partly supported by the Spanish Ministry of Science and Education through project CONSOLIDER INGENIO 2010 CSD2007-00004 "ARES".

\section{References}

[1] R. Apéry. Sur les branches superlinéaires des courbes algébriques. C. R. Acad. Sci. Paris, 222:1198-1200, 1946.

[2] D. E. Dobbs and G. L. Matthews. On a question of Wilf concerning numerical semigroups. International Journal of Commutative Rings, 3(2), 2003.

[3] R. Fröberg, C. Gottlieb, and R. Häggkvist. On numerical semigroups. Semigroup Forum, 35(1):63-83, 1987.

[4] M. Madero-Craven and K. Herzinger. Apery sets of numerical semigroups. Comm. Algebra, 33(10):3831-3838, 2005.

[5] J. C. Rosales, P. A. García-Sánchez, J. I. García-García, and M. B. Branco. Systems of inequalities and numerical semigroups. J. London Math. Soc. (2), 65(3):611-623, 2002. 
[6] J. C. Rosales, P. A. García-Sánchez, J. I. García-García, and M. B. Branco. Numerical semigroups with a monotonic Apéry set. Czechoslovak Math. J., 55(130)(3):755-772, 2005.

[7] N. J. A. Sloane. The on-line encyclopedia of integer sequences.

[8] H. S. Wilf. A circle-of-lights algorithm for the "money-changing problem". Amer. Math. Monthly, 85(7):562-565, 1978. 